\title{
Treating Ulcerative Colitis by Activating Blood Circulation to Dissipate Blood Stasis
}

\author{
Yi Hu${ }^{1}$, Tao $\mathrm{Yu}^{2 *}$ \\ 1Shaanxi University of Chinese Medicine, Xianyang 712046, Shaanxi Province, China \\ ${ }^{2}$ Shaanxi Provincial Hospital of Chinese Medicine, Xi'an 710003, Shaanxi Province, China \\ *Corresponding author: Tao Yu, yt0745@163.com
}

\begin{abstract}
Ulcerative colitis (UC) is a worldwide refractory bowel disease with unclear pathogenesis. Its lesions can lead to colorectal cancer over time. UC is classified as "diarrhea," "dysentery," and other categories in traditional Chinese medicine. Its etiology and pathogenesis in traditional Chinese medicine are complex, but it has been found that blood stasis plays an important role in its occurrence and development. Combined with modern research, the relationship between blood stasis and ulcerative colitis is analyzed in this article along with a discussion on the therapeutic effect of activating blood and removing blood stasis on the disease, aiming to provide new ideas for the treatment of UC.
\end{abstract}

Keywords: Ulcerative colitis; Diarrhea; Dysentery; Activating blood circulation to dissipate blood stasis

Publication date: September 2021; Online publication: September 30, 2021

\section{Introduction}

Ulcerative Colitis (UC) is a refractory intestinal disease caused by the interaction of environmental factors and genetic factors along with the presence of intestinal microorganisms, resulting in intestinal immune imbalance, barrier damage, and continuous inflammatory damage of the intestinal mucosa ${ }^{[1]}$. In recent years, the prevalence of ulcerative colitis in China is increasing gradually; however, there is a lack of effective treatment for this condition in Western medicine. According to the clinical symptoms of UC, UC is classified as "diarrhea," "dysentery," and other diseases. The course of the disease is long, and blood stasis plays an important role in its development, thus activating blood circulation to dissipate blood stasis is an important method to treat UC.

\section{Relationship between blood stasis and ulcerative colitis in traditional Chinese medicine}

In traditional medicine, spleen deficiency is the basis of UC while damp-heat, phlegm turbidity, and blood stasis are the main pathological factors, among which blood stasis runs through the beginning and the end of UC. Ulcerative colitis patients with weak spleen and stomach, endogenous phlegm turbidity, long yu heat, or concurrently with blood stasis poison, the flow of qi and blood to intestinal organs are blocked; damp-heat, phlegm turbidity, other evils, as well as intestinal qi and blood struggle with one another. Heat toxin fumigation of the intestinal organs causes damage to the lipid membrane and blood collaterals, leading to rotting flesh and poison in the blood; and finally, form ulcers. Whatever the etiology that causes poor blood flow such as affect-mind dissatisfaction and liver qi depression, it does not only reverse the spleen and damages both the spleen and stomach, but also affect the smooth flow of blood, forming stasis due to weakness caused by the inability of promoting the operation of blood. The deficiency of yang in the body or long illnesses injures the yang, resulting in the inner generation of yin and coldness; cold evil coagulates 
the meridians, then blood coagulates astringently and runs slowly, resulting in blood stasis. Blood stasis does not disperse; it does not only affect the generation of new blood, but also hinders the distribution of qi and blood. This alternate development finally affects the recovery of patients with UC.

\section{Relationship between ulcerative colitis and hemorheology}

In recent years, many researchers have been observing the hemorheology of UC patients; it has been found that these patients often have high blood viscosity. In a study, Qunlian Lin and other researchers ${ }^{[2]}$ found that blood viscosity, hematocrit, and fibrinogen index were significantly higher among UC patients. The high viscosity of blood causes the flow of blood to be slow, resulting in the formation of microthrombus, and eventually leading to intestinal mucosal capillary occlusion, intestinal mucosal tissue necrosis, as well as ulceration. Ji Qian and other researchers ${ }^{[3]}$ found that patients with UC had significantly higher platelets, MPV (mean platelet volume), and D-dimer compared to those in the control group, suggesting that with UC, the patients' bodies are in a hypercoagulable state and thrombosis is one of the important pathogeneses of UC. In another study, Li Jiang and another researcher ${ }^{[4]}$ found that the levels of D-dimer and fibrinogen in the plasma of patients with ulcerative colitis were significantly higher than those in the control group, and the partial thrombin time was significantly shorter than those in the control group, indicating that patients with UC have high blood coagulability state and secondary hyperfibrinolytic system. The high blood viscosity along with small intestinal microcirculation lumen in UC patients cause blood to remain and silt easily, resulting in capillary occlusion, followed by tissue ischemia and incomplete oxygen supply; in turn, a large amount of oxygen free radicals is produced, damaging the intestinal mucosa and eventually causing ulcers.

Many studies have shown that patients with ulcerative colitis have hypercoagulable and viscous blood, blood stasis and deposition of intestinal microcirculation, slow blood flow in capillaries, as well as the occurrence of thromboembolism, which is consistent with blood stasis in traditional Chinese medicine.

\section{Research on the treatment of $\mathrm{UC}$ by activating blood circulation to dissipate blood stasis}

Pharmacological experiments have shown that the blood-activating and stasis-resolving drug could promote metabolism, improve microcirculation, fight thrombosis, remove inflammatory products and cytotoxicity, promote tissue repair, as well as benefit ulcer healing. Clinical studies have shown that angelica injection and Salvia miltiorrhiza injection ${ }^{[5,6]}$ can reduce D-dimer, inhibit platelet aggregation, reduce blood viscosity and hypercoagulable states, effectively improve blood circulation, and promote intestinal mucosal repair. In addition, it can also remove oxygen free radicals and reduce the damage of inflammatory reaction to intestinal cells. Yao Gong and another researcher ${ }^{[7]}$ treated a group of UC patients with perfusion fluid, comprising of Salvia beverage, Radix Paeoniae Rubra, Hirudo, Radix Pulsatillae, and other drugs. The blood rheology and microcirculation of these patients effectively improved, indicating that promoting blood circulation and removing blood stasis can improve blood flow and promote ulcer healing. Pharmacological studies have shown that Radix Paeoniae Rubra can reduce the activity of coagulation factors, prevent the aggregation of red blood cells and platelets, increase the content of nitric oxide (NO), promote vascular relaxation, as well as aid in achieving anti-thrombotic effect ${ }^{[8]}$. The treatment of ulcerative colitis by activating blood circulation and removing blood stasis, according to syndrome differentiation, is able to remove blood stasis and rotting flesh, produce new blood, as well as promote the healing of colonic mucosal ulcer.

\section{Conclusion}

Although the etiology and pathogenesis of ulcerative colitis are unclear, patients are often in pathological 
states, such as having high blood viscosity and intestinal microcirculation disorders. Promoting blood circulation and removing blood stasis can improve hemorheology, reduce inflammatory reactions, as well as promote tissue repair and ulcer healing. Therefore, activating blood circulation to dissipate blood stasis is an important rule in the treatment of UC. However, in clinical practice, the method of activating blood circulation to dissipate blood stasis should be used according to syndrome differentiation. At the same time, it is necessary to take into account the elimination of evil and not forgetting to support healthy energy as well as to avoid being obsessed with one law while forgetting the original.

\section{Disclosure statement}

The author declares no conflict of interest.

\section{References}

[1] Zhang S, Shen H, Zheng K, Ye B, 2017, Ulcerative Colitis Expert Consensus on TCM Diagnosis and Treatment. China Journal of Traditional Chinese Medicine and Pharmacy, 32(08): 3585-3589.

[2] Lin Q, Huang F, Cai S, et al., 2002, Hemorheological Observation of Patients with Damp Heat Syndrome of Chronic Nonspecific Colitis. Traditional Chinese Medicine Journal, 4(06): 17-18

[3] Qian J, Dong S, Chen G, 2016, Clinical Significance of Platelet Count, Mean Platelet Volume and D-dimer Detection in Patients with Ulcerative Colitis. Journal of Nantong University (Medical Science), 36(01): 72-74.

[4] Jiang L, Xiao C, 2012, Clinical Significance of Changes in Coagulation Function and C-reactive Protein in Patients with Ulcerative Colitis. Journal of Hunan Normal University (Medical Sciences), 22(2): 91-93.

[5] Zhang J, Zhang Y, Hao Y, et al., 2008, Effect and Significance of Salvia Miltiorrhiza Injection on D-dimer Content in Patients with Ulcerative Colitis. Modern Journal of Integrated Traditional Chinese and Western Medicine, (32): 4968-4969.

[6] Liu S, Dong W, Liu S, et al., 2003, Effect of Angelica Injection on Platelet Function in Patients with Ulcerative Colitis and Its Significance. Journal of Chinese Physician, (07): 904-906.

[7] Gong Y, Wang Y, 1997, Observation on the Efficacy of Clearing Heat and Removing Blood Stasis in the Treatment of 36 Cases of Ulcerative Colitis. Clinical Journal of Anhui Traditional Chinese Medicine, (02): 67-68

[8] Wu L, Wang Z, He K, et al., 2021, Study on Chemical Constituents and Pharmacological Effects of Radix Paeoniae Lactiflora. Chinese Journal of Experimental Traditional Medical Formulae, : 1-30. 